\title{
A NOTE ON EXPONENTIAL INTEGRABILITY AND POINTWISE ESTIMATES OF LITTLEWOOD-PALEY FUNCTIONS
}

\author{
MARK LECKBAND
}

(Communicated by J. Marshall Ash)

\begin{abstract}
Let $T f$ denote any one of the usual classical or generalized Littlewood-Paley functions. This paper derives a BLO norm estimate for $(T f)^{2}$ and a pointwise estimate for $T f$.
\end{abstract}

\section{INTRODUCTION}

In this paper we will derive a BLO norm estimate and a pointwise inequality for $T f$ being any one of the usual classical and generalized Littlewood-Paley functions.

Let $f$ belong to $L^{\infty}$. We shall obtain (Theorem 1) that $T f$ satisfies

$$
\left\|(T f)^{2}\right\|_{\mathrm{BLO}} \leq C\|f\|_{\infty}^{2}
$$

This kind of result can be found in [1], and by duality in [3] and [4]. However our result is motivated by the distribution inequalities of Murai and Uchiyama [8], where $T f$ is the Lusin area integral.

A function $f$ is said to belong to BLO if

$$
\int_{Q} f(x)-\inf _{Q}(f) d x \leq C|Q|,
$$

for any cube $Q$ of $R^{n}$. The John-Niremberg lemma for BMO, where $\inf _{Q}(f)$ is replaced by ave $e_{Q}(f)$, still holds for BLO with a usual proof, see [6], requiring easy modifications. Thus (1.1) implies $T f$ is exponentially square integrable in the sense of

$$
\int_{Q} \exp \left\{\frac{C_{1}}{\|f\|_{\infty}^{2}}\left[(T f(x))^{2}-\inf _{Q}(T f)^{2}\right]\right\} d x \leq C_{2}|Q| .
$$

Received by the editors January 16, 1989 and, in revised form, July 24, 1989.

1980 Mathematics Subject Classification (1985 Revision). Primary 42B25. 
Some understanding of the difference between BLO and BMO can be found by looking at the class of Calderon-Zygmund singular integral operators. Consider the simplest example, the Hilbert transform $H f$. For $f$ in $L^{\infty}$, the function $H f$ belongs to $\mathrm{BMO}$, but $H f$ will not belong to $\mathrm{BLO}$ in general. Yet the maximal Hilbert transform, though pointwise larger, maps $L^{\infty}$ into BLO, see Lemma 1. of [7]. Since the proof of this result requires only an inequality known as a good- $\lambda$ inequality, we can state that this trait is characteristic of the class of $\mathrm{C}-\mathrm{Z}$ operators. We may infer that maximizing a singular integral operator creates a less varying operator that is insignificantly larger. We should also note that (1.1) involves the square of the L-P function and what we have just said about a $\mathrm{C}-\mathrm{Z}$ operator does not hold for the square of the operator, for example, $(H f)^{2}$ does not belong to BMO.

Littlewood-Paley functions represent an example of what are called vectorvalued $C-Z$ singular integral operators, this is discussed in [6]. We can see that (1.1) really shows two distinctions between $\mathrm{L}-\mathrm{P}$ functions and $\mathrm{C}-\mathrm{Z}$ singular integral operators. The former are significantly smaller, since the singularities are no worse than $(\log (1 / t))^{1 / 2}$ verses $\log (1 / t)$ for bounded functions, and the former vary less. This is at odds with the usual assumption that vector-valued singular integral operators are just as bad as singular integral operators.

Our second result is motivated by the pointwise inequalities of Calderon and Torchinsky [2], Chanillo and Wheeden [4], and Stein [9]. Specifically we show

$$
T f(x) \leq C g_{\mu}^{*}(f)(x), \quad 1<\mu<(n+2) / n,
$$

where $g_{\mu}^{*}(f)$ is the classical function defined in [9]. The generalized L-P functions are usually, and will be defined here using Schwartz functions $\theta$ in place of the Poisson kernel $P$. Many variations of (1.4) are known, see [2, 4, 9], even with the L-P functions on both sides defined by using kernels $\theta_{1}$ and $\theta_{2}$. However the generality of (1.4) with $g_{\mu}^{*}(f)$ on the dominant side seems to be new.

\section{Preliminaries}

We now set up our notation and definitions. For $x$ in $R^{n}, r>0$ and $\alpha>0$, let

$$
\begin{aligned}
& B(x, r)=\left\{y \in R^{n}:|x-y|<r\right\} \\
& \Gamma(x, \alpha)=\left\{(y, t) \in R_{+}^{n+1}:|x-y|<\alpha t\right\}
\end{aligned}
$$

The symbol $\theta$ will always be a Schwartz function with $\int \theta=0$. We will denote the Poisson kernel $C_{n} t /\left(t^{2}+|x|^{2}\right)^{(n+1) / 2}$ by $P_{t}(x)$ [9], where the constant $C_{n}$ is chosen so that $\int P=1$. The constant of the Fourier transform is chosen so that $\widehat{P}_{t}(\zeta)=e^{-|\zeta|}$. 
Definition 1. Let $g(f), S(f, \alpha)$, and $g_{\mu}^{*}(f)$ be the same Littlewood-Paley functions defined in Stein [9]. We define their generalizations as

$$
\begin{aligned}
g(f, \theta)(x) & =\left(\int_{0}^{\infty}\left|f * \theta_{t}(x)\right|^{2} \frac{d t}{t}\right)^{1 / 2}, \\
s(f, \theta, \alpha)(x) & =\left(\iint_{\Gamma(x, \alpha)}\left|f * \theta_{t}(y)\right|^{2} \frac{d y d t}{t^{n+1}}\right)^{1 / 2}, \\
g_{\mu}^{*}(f, \theta)(x) & =\left(\iint_{R_{+}^{n+1}}\left(\frac{t}{t+|x-y|}\right)^{n \mu}\left|f * \theta_{t}(y)\right|^{2} \frac{d y d t}{t^{n+1}}\right)^{1 / 2},
\end{aligned}
$$

where $\mu>1$.

In our general formulas $T f$ will denote any one of the L-P functions mentioned in Definition 1. The functions $T_{r} f$ and $T_{r}^{\prime} f$ will be the same with the integration of the $t$ variable restricted to $(0, r)$ and $[r, \infty)$, respectively. For constants that do not depend upon the function $f$ we shall use the letter $C$ that may change from line to line. We shall complete our preliminaries by listing a standard result whose proof may be found in [6].

Lemma 1. Let $f$ belong to $\mathrm{BMO}$. Then

$$
\left|\nabla_{x} f * P_{t}(x)\right|^{2} t d x d t \text { and }\left|f * \theta_{t}(x)\right|^{2} d x d t / t
$$

are Carleson measures. In particular, for $f$ in $L^{\infty}$, the constant is $C\|f\|_{\infty}^{2}$.

\section{BLO ESTIMATE}

In this section we prove the first main result which we call Theorem 1. Our argument will develop along lines similar to that of [7]. To control $T f$ we use the decomposition $(T f)^{2}=\left(T_{r} f\right)^{2}+\left(T_{r}^{\prime} f\right)^{2}$. Our first lemma states $\left(T_{r}^{\prime} f\right)^{2}$ is Lipschitzian.

Lemma 2. Let $f$ belong to $L^{\infty}$. Then for $x, z \in R^{n}$,

$$
\left|\left(T_{r}^{\prime} f(x)\right)^{2}-\left(T_{r}^{\prime} f(z)\right)^{2}\right| \leq C\|f\|_{\infty}^{2}\left|\frac{x-z}{r}\right| .
$$

Proof. The case when $T f$ is $S(f, \alpha)$ or $S(f, \theta, \alpha)$ proceeds exactly as Lemma 3.1 of [7]. Easiest is the case when $T f$ is $g(f)$ or $g(f, \theta)$.

$$
\begin{aligned}
& \left.\mid\left(g_{r}^{\prime}(f), \theta\right)(x)\right)^{2}-\left(g_{r}^{\prime}(f, \theta)(z)\right)^{2} \mid \\
& \quad \leq \int_{r}^{\infty}\left|f * \theta_{t}(x)-f * \theta_{t}(z)\right|\left|f * \theta_{t}(x)+f * \theta_{t}(z)\right| \frac{d t}{t}, \\
& \quad \leq C\|f\|_{\infty}^{2} \int_{r}^{\infty} \frac{|x-z|}{t^{2}} d t=C\|f\|_{\infty}^{2} \frac{|x-z|}{r} .
\end{aligned}
$$

Clearly $T f=g(f)$ has the same proof.

Now consider $T f=g_{\mu}^{*}(f, \theta)$ and note that $g_{\mu}^{*}(f)$ will have the same proof.

$$
\begin{aligned}
& \left|\left(g_{\mu}^{*}(f, \theta)(x)\right)^{2}-\left(g_{\mu}^{*}(f, \theta)(z)\right)^{2}\right| \\
& \quad \leq C\|f\|_{\infty}^{2} \int_{R^{n}} \int_{r}^{\infty}\left|\left(\frac{t}{t+|x-y|}\right)^{n \mu}-\left(\frac{t}{t+|z-y|}\right)^{n \mu}\right| \frac{d t d y}{t^{n+1}} .
\end{aligned}
$$


Observe that,

$$
\left|\left(\frac{1}{t+|x-y|}\right)^{n \mu}-\left(\frac{1}{t+|z-y|}\right)^{n \mu}\right| \leq C \frac{|x-z|(t+|x-y| \vee|z-y|)^{n \mu-1}}{(t+|x-y|)^{n \mu}(t+|z-y|)^{n \mu}}
$$

We do our estimates according to different cases.

I. First consider $t>|x-y| \mathrm{V}|z-y|$. Using (3.3) over this region of integration, we obtain

$$
A_{\mathrm{I}} \leq C\|f\|_{\infty}^{2} \int_{r}^{\infty} \int_{R^{n} \cap\{y: t>|x-y| \vee|z-y|\}} \frac{|x-z| t^{2 n \mu-1}}{t^{2 n \mu}} \frac{d y d t}{t^{n+1}} \leq C\|f\|_{\infty}^{2} \frac{|x-z|}{r} .
$$

II. Now consider $|x-y| \geq|y-z| \geq t$. Again using (3.3) we get

$$
A_{\mathrm{II}} \leq C\|f\|_{\infty}^{2} \int_{R^{n} \backslash B(x, r) \cup B(z, r)} \int_{r}^{|y-z|} \frac{|x-z||x-y|^{n \mu-1} t^{n \mu}}{|x-y|^{n \mu}|y-z|^{n \mu}} \frac{d t d y}{t^{n+1}} .
$$

For $n \mu>n$ we have,

$$
\begin{aligned}
& \leq C\|f\|_{\infty}^{2} \int_{R^{n} \backslash B(x, r) \cup B(z, r)} \frac{|x-z|}{|x-y||y-z|^{n}} d y \\
& \leq C\|f\|_{\infty}^{2}|x-z| \int_{r}^{\infty} \frac{1}{p^{2}} d p, \\
& \leq C\|f\|_{\infty}^{2} \frac{|x-z|}{r} .
\end{aligned}
$$

It may be of interest to note that for $1>n-n \mu>0$ the same estimate can be obtained.

III. Now we consider $|x-y| \geq t>|y-z|$ which will complete the proof. Using (3.3) we have

$$
\begin{aligned}
A_{\mathrm{III}} & \leq C\|f\|_{\infty}^{2} \int_{r}^{\infty} \int_{|y-z|<t} \frac{|x-z||x-y|^{n \mu-1} t^{n \mu}}{|x-y|^{n \mu} t^{n \mu}} \frac{d y d t}{t^{n+1}} \\
& \leq C\|f\|_{\infty}^{2} \int_{r}^{\infty} \frac{|x-z| t^{n}}{t^{n+2}} d t \leq C\|f\|_{\infty}^{2} \frac{|x-z|}{r} .
\end{aligned}
$$

Lemma 3. Let $f$ belong to $L^{\infty}$. Then

$$
\int_{B(z, r)}\left(T_{r} f(x)\right)^{2} d x \leq C\|f\|_{\infty}^{2}|B(z, r)| \text {. }
$$

Proof. We shall just do the case for $T f=g_{\mu}^{*}(f, \theta)$. The other cases are simpler and more direct.

$$
\begin{aligned}
\int_{B(z, r)}\left(T_{r} f(x)\right)^{2} d x & =\int_{0}^{r} \int_{B(z, r)} \int_{R^{n}}\left(\frac{t}{t+|x-y|}\right)^{n \mu}\left|f * \theta_{t}(y)\right|^{2} \frac{d y d x d t}{t^{n+1}}, \\
& =B_{\mathrm{I}}+B_{\mathrm{II}}+B_{\mathrm{III}} .
\end{aligned}
$$

The above quantities are integrals where the $y$-integration is over $|x-y|<$ $t, t \leq|x-y|<r$ and $r \leq|x-y|$, respectively. 
For $|x-y|<t$ we have $t /(t+|x-y|) \leq C$. Thus with a change in the order of the $x$ and $y$ integration, and then carrying out the $x$-integration, we have

$$
B_{1} \leq C \int_{0}^{r} \int_{B(z, C r)}\left|f * \theta_{t}(y)\right|^{2} \frac{d y d t}{t} \leq C\|f\|_{\infty}^{2}|B(z, r)|,
$$

where we have used Lemma 1 for the last inequality.

For $t \leq|x-y|<r$ we have $t /(t+|x-y|) \leq C t /|x-y|$. Again after changing the order of integration we have

$$
B_{\mathrm{II}} \leq C \int_{0}^{r} \int_{B(z, C r)} t^{n \mu}\left(\frac{1}{t^{n \mu-1}}\right)\left|f * \theta_{t}(y)\right|^{2} \frac{d y d t}{t^{n+1}} \leq C\|f\|_{\infty}^{2}|B(z, r)| .
$$

The last inequality is by Lemma 1 .

To do the last case, $r \leq|x-y|$, we begin by writing $R^{n} \backslash B(z, r)=\bigcup A_{k}$, where $A_{k}$ is the annulus centered at $z$ with inside radius $r 2^{k}$ and outside radius $r 2^{k+1}, k=0,1,2,3, \ldots$ Using this decomposition and

$$
\left(\frac{t}{t+|x-y|}\right) \leq \frac{C t}{r \vee|x-y|}
$$

then

$$
\begin{aligned}
B_{\mathrm{III}} & \leq C \sum \int_{0}^{r} \int_{B(z, r)} \int_{A_{k}} \frac{t^{n \mu}}{(|x-y| \vee r)^{n \mu}}\left|f * \theta_{t}(y)\right|^{2} \frac{d y d x d t}{t^{n+1}}, \\
& \leq C \sum \frac{1}{\left(r 2^{k}\right)^{n \mu}} \int_{0}^{r} \int_{B(z, r)} \int_{B\left(z, r 2^{k+1}\right)} t^{n \mu-n}\left|f * \theta_{t}(y)\right|^{2} \frac{d y d x d t}{t} .
\end{aligned}
$$

Using $t^{n \mu-n} \leq r^{n \mu-n}$ and Lemma 1 we derive

$$
\leq C\|f\|^{2} \sum \frac{\left(r^{n}\right)\left(r^{n \mu-n}\right)\left(r 2^{k+1}\right)^{n}}{\left(r 2^{k}\right)^{n \mu}} \leq C\|f\|_{\infty}^{2}|B(z, r)| .
$$

The proof is complete.

Now we come to the result of this section.

Theorem 1. Let $f$ belong to $L^{\infty}$. Then

$$
\left\|(T f)^{2}\right\|_{\mathrm{BLO}} \leq C\|f\|_{\infty}^{2} .
$$

Proof. Observe that $(T f)^{2}=\left(T_{r} f\right)^{2}+\left(T_{r}^{\prime} f\right)^{2}$ and let $Q$ be any cube of $R^{n}$. Let $z_{0}$ be the center of $Q$ and let half the diagonal be $r$.

$$
\begin{aligned}
\int_{Q}(T f(x))^{2}-\inf _{Q}(T f)^{2} d x \leq & \int_{Q}\left(T_{r}^{\prime} f(x)\right)^{2}-\inf _{Q}\left(T^{\prime} f\right)^{2} d x \\
& +\int_{B\left(z_{0}, r\right)}\left(T_{r} f(x)\right)^{2} d x, \\
\leq & C\|f\|_{\infty}^{2}|Q|,
\end{aligned}
$$

by Lemmas 2 and 3, respectively. 


\section{Pointwise estimate}

In establishing the pointwise estimate (Theorem 2 ) we begin by proving two fairly technical lemmas. The first lemma below is ultimately needed for decay estimates on $\tau * P(x)$, where $\tau$ is a Schwartz function. Trouble arises since $P^{\wedge}(\zeta)=-e^{|\zeta|}$ misses being a Schwartz function because of nondifferentiability at 0 .

Lemma 4. Let $|D|$ be the operator defined by $(|D| \tau)^{\wedge}(\zeta)=|\zeta| \tau^{\wedge}(\zeta)$, for Schwartz functions $\tau$. Then for $0<\beta<1$,

$$
|| D|\tau(x)| \leq \frac{C_{\beta}}{(1+|x|)^{n+\beta}} .
$$

Proof. We prove this lemma by modifying many of the ideas found on page 133 of Stein [9]. To begin

$$
|\zeta|=\frac{1}{2 \pi} \frac{\left(4 \pi^{2}|\zeta|^{2}\right)^{1 / 2}}{\left(1+4 \pi^{2}|\zeta|^{2}\right)^{1 / 2}} \cdot\left(1+4 \pi^{2}|\zeta|^{2}\right)^{1 / 2}
$$

The second factor $\left(1+4 \pi^{2}|\zeta|^{2}\right)^{1 / 2}$ maps Schwartz functions to Schwartz functions and so we consider it no longer. We decompose the first factor using

$$
(1-t)^{1 / 2}=1+\sum A_{m} t^{m}, \quad A_{m}=(-1)^{m}\left(\begin{array}{c}
1 / 2 \\
m
\end{array}\right) .
$$

That is

$$
\begin{aligned}
\frac{\left(4 \pi^{2}|\zeta|^{2}\right)^{1 / 2}}{\left(1+4 \pi^{2}|\zeta|^{2}\right)^{1 / 2}} & =1+\sum A_{m}\left(1+4 \pi^{2}|\zeta|^{2}\right)^{-m} \\
& =1+\sum A_{m} G_{2 m}^{-}(\zeta)
\end{aligned}
$$

where $G_{2 m}$ is the kernel of the Bessel potential [9]. Note that for large $m, A_{m}$ is of constant sign and

$$
A_{m} \sim \frac{\Gamma(m-1+1 / 2)}{\Gamma(m+1)} \sim m^{-3 / 2}
$$

using $\Gamma(m) \sim \sqrt{2 \pi m} m^{m} e^{-m}$. We must obtain a decay estimate that does not overpower the coefficients $A_{m}$. Note that we may restrict ourselves to $|x|>1$ by Lemma 2(i) of [9]. Also it is shown in [9] that $G_{2 m}(x) \sim O\left(e^{-|x| / 2}\right)$, but the constant increases too quickly with $m$. We begin anew with the following identity.

$$
(4 \pi)^{m} \Gamma(m) G_{2 m}(x)=\int_{0}^{\infty}\left(e^{-|x|^{2} \pi / \delta} \delta^{-\imath-n / 2}\right) e^{-\delta / 4 \pi} \delta^{m+\alpha} \frac{d \delta}{\delta} .
$$

The factor within the parenthesis equals $\exp \left\{-|x|^{2} \pi / \delta-(\alpha+n / 2) \ln (\delta)\right\}$, and has a maximum value of

$$
e^{(\alpha+n / 2)} /\left[\frac{2 \pi}{(2 \alpha+n)}|x|^{2}\right]^{\alpha+n / 2}
$$


that occurs at

$$
\delta_{0}=\frac{2 \pi|x|^{2}}{(2 \alpha+n)}
$$

With $|x|>1$,

$$
(4 \pi)^{m} \Gamma(m) G_{2 m}(x) \leq \frac{C_{\alpha}}{(1+|x|)^{n+2 \alpha}} \int_{0}^{\infty} e^{-\delta / 4 \pi} \delta^{m+\alpha} \frac{d \delta}{\delta} .
$$

or

$$
G_{2 m}(x) \leq \frac{C_{\alpha}}{(1+|x|)^{n+2 \alpha}} \frac{\Gamma(m+\alpha)}{\Gamma(m)}<\frac{C_{\alpha}}{(1+|x|)^{n+2 \alpha}} m^{\alpha} .
$$

Letting $\beta=2 \alpha<1$ and the above applied to (4.1) gives us

$$
\left[\frac{\left(4 \pi^{2}|\zeta|^{2}\right)^{1 / 2}}{\left(1+4 \pi^{2}|\zeta|^{2}\right)^{1 / 2}}\right]^{-}(x) \leq \frac{C_{\beta}}{(1+|x|)^{n+\beta}} .
$$

Thus $|D| \dot{\tau}(x)$ is a Schwartz function convolved with (4.2). The lemma now follows.

The next lemma is motivated by Lemmas 1.4 and 1.8 of Chanillo and Wheeden [4] which in turn is a modification of the methods of Stromberg and Torchinsky [10].

Lemma 5. Let $a>0$ and $M$ (large) $>0$. Then for $a$ Schwartz function $f$ and $1<\mu<(n+2) / n$,

$$
\left|\frac{f * \theta_{s}(y)}{s}\right|^{2} \leq C \int_{0}^{a s} \int_{R^{n}}\left|\nabla_{z}\left(f * P_{t}\right)(z)\right|^{2} \frac{(t / s)^{M}}{\left(1+\frac{|y-z|}{t}\right)^{n \mu}} \frac{d z d t}{t^{n+1}} .
$$

Proof. Our proof of this lemma begins as a repetition of the proof of Lemma 1.8 of [4]. To make what follows comprehensible we shall put in all the details rather than just paraphrasing and jumping into the middle.

First, for $0<\varepsilon<\delta<a$, there exists a $n^{-}(\zeta) \in C_{0}^{\infty}$ such that supp $n^{-}$is contained in $\{\varepsilon<|\zeta|<\delta\}, n^{-}>0$ and for $\zeta=0$

$$
\int_{0}^{\infty} p^{\widehat{A}}(t \zeta) \hat{n}(t \zeta) \frac{d t}{t}=1
$$

To see this, choose $\Phi(t)$ in $C_{0}^{\infty}, \Phi>0$, such that supp $\Phi$ is contained in the interval $(\varepsilon, \delta)$. Set

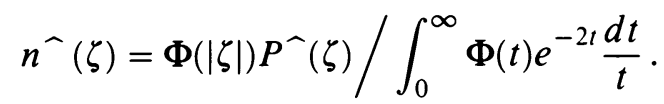

Since 0 does not belong to the support of $\Phi$ we have $n^{-}$with the desired properties.

Given $a>0$ define

$$
h(\zeta)=1-\int_{0}^{a} P^{-}(t \zeta) n^{\widehat{ }}(t \zeta) \frac{d t}{t} .
$$


Then $h$ is $C^{\infty}, h=1$ near 0 , and for $\zeta$ not 0 ,

$$
h(\zeta)=\int_{a}^{\infty} P^{\wedge}(t \zeta) n^{\wedge}(t \zeta) \frac{d t}{t} .
$$

Moreover $h$ has compact support since $n^{\wedge}$ does.

Now choose a $C^{\infty}$ function $\tau$ with $\operatorname{supp} \tau$ contained in the interval $(\varepsilon, \delta)$ so that $\int_{0}^{\infty} \tau(t) / t d t=1$. Then

$$
h(\zeta)=\int_{0}^{a} h(\zeta) \frac{\tau(t)}{P^{\wedge}(t \zeta)} P^{\wedge}(t \zeta) \frac{d t}{t} .
$$

Combining (4.4) and (4.5) we have

$$
\int_{0}^{a} P^{\wedge}(t \zeta)\left[n^{\wedge}(t \zeta)+\frac{h(\zeta) \tau(t)}{P^{\wedge}(t \zeta)}\right] \frac{d t}{t}=1 .
$$

Set $\sigma^{\wedge}(\zeta, t)=n^{\wedge}(\zeta)+h(\zeta / t) \tau(t) / P^{\wedge}(\zeta)$.

Using the above identity, we have

$$
\begin{aligned}
& \frac{\theta^{\wedge}(s \zeta)}{s}=\frac{\theta^{\frown}(s c)}{s} \int_{0}^{a s} P^{\wedge}(t \zeta) \sigma^{\wedge}(t \zeta, t / s) \frac{d t}{t}, \\
& =\sum_{i=1}^{n} \int_{0}^{a s} \zeta_{i} P^{\wedge}(t \zeta) \frac{\zeta_{i}}{s|\zeta|^{2}} \theta^{\wedge}(s \zeta) \sigma^{\wedge}(t \zeta, t / s) \frac{d t}{t}, \\
& =\sum_{i=1}^{n} \int_{0}^{a s}\left[\frac{\partial}{\partial y_{i}} P_{t}\right]-(\zeta) T_{i}^{\curlywedge}(t \zeta, t / s) \frac{d t}{t},
\end{aligned}
$$

and so,

$$
\frac{f * \theta_{s}(y)}{s}=\sum_{i=1}^{n} \int_{0}^{a s} \int_{R^{n}}\left[\frac{\partial}{\partial z_{i}}\left(f * P_{t}\right)(z)\right] T_{i}\left(\frac{y-z}{t}, \frac{t}{s}\right) \frac{d z d t}{t^{n+1}},
$$

where $T_{i}^{\wedge}(\zeta, t)=\theta^{\wedge}(\zeta / t)\left(\zeta_{i} / t\right) /|\zeta / t|^{2} \cdot\left[n^{\wedge}(\zeta)+e^{|\zeta|} \tau(t) h(\zeta / t)\right]$.

We now establish, for $0<t<a$, the estimate

$$
\left|T_{i}(x, t)\right|<C_{\beta} \frac{t^{M}}{(1+|x|)^{n+\beta}}, \quad 0<\beta<1,
$$

where the dependence of $\beta$ arises from Lemma 4. Fix $i$ and denote $g^{\wedge}(\zeta / t)=$ $\left(\zeta_{i} / t /|\zeta / t|^{2}\right) \cdot \theta^{\wedge}(\zeta / t)$. Since $\theta^{-}(0)=0$ and $\theta^{\wedge}$ is a Schwartz function, we have that $g^{\wedge}$ is a Schwartz function. Using $e^{|\zeta|}=\cosh (|\zeta|)+\sinh (|\zeta|)$, then

$$
\begin{aligned}
T_{i}^{\wedge}(\zeta, t)= & g^{\wedge}(\zeta / t) n^{\wedge}(\zeta)+g^{\wedge}(\zeta / t) \cosh (|\zeta|) \tau(t) h(\zeta / t) \\
& +|\zeta|\left[g^{\wedge}(\zeta / t) \frac{\sinh (|\zeta|)}{|\zeta|} \tau(t) h(\zeta / t)\right], \\
= & A^{\wedge}(\zeta, t)+B^{\wedge}(\zeta, t)+|\zeta| C^{\curlywedge}(\zeta, t) .
\end{aligned}
$$

First we have,

$$
\left|D_{\zeta}^{\prime \prime}\left(A^{\curlyvee}(\zeta, t)\right)\right| \leq \frac{C}{t^{|\alpha|}} \frac{X_{\text {supp } n^{\wedge}}(\zeta)}{(1+|\zeta| / t)^{K}}, \quad 0<t<a .
$$


Using $|\zeta|>\varepsilon>0$ in $\operatorname{supp} n^{\wedge}$, then

$$
\left|D_{\zeta}^{\alpha}\left(A^{\curlyvee}(\zeta, t)\right)\right| \leq C t^{K-|\alpha|} X_{\{|\zeta|<\delta\}}(\zeta) .
$$

Hence $|A(x, t)| \leq C t^{M} /(1+|x|)^{N}$, where $M$ and $N$ can be taken as large as desired.

To estimate $B(x, t)$ observe that $B^{\frown}(\zeta, t)$ is a Schwartz function with respect to the $\zeta$ variable and $\operatorname{supp}(\tau)$ is contained in the interval $(\varepsilon, \delta)$. Hence $|B(x, t)| \leq C t^{M} /(1+|x|)^{N}$.

Finally consider $C^{\wedge}(\zeta, t)$. The above statements about $B^{\wedge}(\zeta, t)$ also apply to $C^{\wedge}(\zeta, t)$. Thus by Lemma 4 ,

$$
\left|\left[|\zeta| C^{\wedge}(\zeta, t)\right]^{\vee}(x)\right| \leq C_{\beta} t^{M} /(1+|x|)^{n+\beta}, \quad 0<\beta<1 .
$$

From these estimates we now have

$$
\left|\frac{f * \theta_{s}(y)}{s}\right| \leq C_{\beta} \int_{0}^{a s} \int_{R^{n}}\left|\nabla_{z} f * P_{t}(z)\right| \frac{(t / s)^{M}}{\left(1+\frac{|y-z|}{t}\right)^{n+\beta}} \frac{d z d t}{t^{n+1}} .
$$

Decomposing $n+\beta=n \mu / 2+[n+\beta-n \mu / 2]$, we may choose a $\beta$ close enough to 1 so that the conclusion of Lemma 5 is derived by using Schwartz's inequality. The restriction of $\beta<1$ gives us the restriction of $\mu<(n+2) / n$ in order to do this last step.

We now come to the second result of this paper.

Theorem 2. Let $1<\mu<(n+2) / n$. Then

$$
T f(x) \leq C g_{\mu}^{*}(f)(x),
$$

where $T$ is any one of the operators of Definition 1 with matching index $\mu$ when $T f=g_{\mu}^{*}(f, \theta)(x)$.

Proof. The case when $T f=g(f, \theta)$ is very easy using Lemma 5. The cases when $T f=g(f)$ and $S(f, \alpha)$ are done in Stein [9]. The case when $T f=$ $S(f, \theta, \alpha)$ proceeds exactly as the final argument of Lemma 1.4 of [4]. Thus we shall restrict ourselves to the case $T f=g_{\mu}^{*}(f, \theta), 1<\mu<(n+2) / n$.

Using Lemma 5 and reversing the order of integration of the $s$ and $t$ variables, we have

$$
\begin{aligned}
& \left|g_{\mu}^{*}(f, \theta)(x)\right|^{2} \\
& \quad \leq C \iint_{R_{+}^{n+1}}\left|\nabla_{z}\left(f * P_{t}\right)(z)\right|^{2}\left[\int_{R^{n}} \int_{t / a}^{\infty} \frac{(t / s)^{M} s^{1-n} t^{-2}}{\left(1+\frac{|y-x|}{s}\right)^{n \mu}\left(1+\frac{|y-z|}{t}\right)^{n \mu}} d s d y\right] \frac{d z d t}{t^{n-1}} .
\end{aligned}
$$

To complete the proof we must show $[\cdots]<[t /(t+|x-z|)]^{n \mu}$. To do this first let $u=x-z$ and the integral inside the brackets becomes less than or equal to

$$
C \int_{R^{n}} \int_{t / a}^{\infty} \frac{(t / s)^{1 \mu} s^{n \mu-n+1} t^{n \mu-2}}{\left(s^{n \mu}+|y|^{n \mu}\right)\left(t^{n \mu}+|u-y|^{n \mu}\right.} d s d y \text {. }
$$




\section{Observe}

$$
\begin{aligned}
& \frac{1}{\left(s^{n \mu}+|y|^{n \mu}\right)\left(t^{n \mu}+|u-y|^{n \mu}\right)}, \\
& \quad=\frac{1}{\left(s^{n \mu}+t^{n \mu}+|y|^{n \mu}+|u-y|^{n \mu}\right)}\left[\frac{1}{s^{n \mu}+|y|^{n \mu}}+\frac{1}{t^{n \mu}+|u-y|^{n \mu}}\right] \\
& \quad \leq C\left(\frac{1}{t+|u|}\right)^{n \mu}[\cdots+\cdots] .
\end{aligned}
$$

Now we have the following integrals with estimates.

$$
\int \frac{1}{s^{n \mu}+|y|^{n \mu}} d y \leq C / s^{n \mu-n} \text { and } \int_{t / a}^{\infty}(t / s)^{M} s t^{n \mu-2} d s \leq C t^{n \mu} .
$$

Also

$$
\int \frac{1}{t^{n \mu}+|u-y|^{n \mu}} d y<C / t^{n \mu-n} \text { and } \int_{t / a}^{\infty}(t / s)^{M} s^{n \mu-n+1} t^{n-2} d s<C t^{n \mu} \text {. }
$$

Thus (4.6) is less than equal to $[t /(t+|u|)]^{n \mu}$ and the proof is complete.

\section{REFERENCES}

1. R. Banuelos and C. N. Moore, Sharp estimates for the nontangential maximal function and the Lusin area function, preprint.

1. A. P. Calderon and A. Torchinsky, Parabolic maximal functions associated with a distribution, Adv. in Math. 16 (1975), 1-64.

3. S. Y. A. Chang, J. Wilson and T. Wolff, Some weighted norm inequalities concerning the Schrodinger operators, Comment. Math. Helv. (to appear).

4. S. Chanillo and R. L. Wheeden, Some weighted inequalities for the area integral, Indiana Univ. Math. J. 36 (1987), 277-294.

5. R. Fefferman, R. Gundy, R. F. Silverstein, and E. M. Stein, Inequalities for ratios of functionals of harmonic functions, Proc. Nat. Acad. Sci. U.S.A. 79 (1982), 7985-7960.

6. J.-L. Journe, Calderon-Zygmund operators, pseudo-differential operators and the Cauchy integral of Calderon, Springer-Verlag, Lecture Notes in Math., vol. 994.

7. M. A. Leckband, Structure results on the maximal Hilbert transform and two-weight norm inequalities, Indiana Univ. Math. J. (2) 34 (1985), 259-275.

8. T. Murai and A. Uchiyama, Good- $\lambda$ inequalities for the area integral and the nontangential maximal function, Studia Math. 83 (1986), 251-262.

9. E. M. Stein, Singular integrals and differentiability properties of functions, Princeton Univ. Press, Princeton, NJ, 1970.

10. O. Stromberg and A. Torchinsky, Weighted Hardy spaces, Lecture Notes in Math., vol. 1381, Springer-Verlag.

Florida International University, Department of Mathematics, Miami, Florida, 33199 